\section{Evolução espaço-temporal do sobrepeso e da obesidade em adolescentes masculinos brasileiros, 1980 a 2005}

\author{
Temporal evolution of overweight and obesity \\ among Brazilian male adolescents, 1980-2005
}

\begin{abstract}
Analysis of the temporal trend in obesity shows the increasing risk posed by this condition worldwide. This article aims to verify and compare the prevalence rates for overweight and obesity in male adolescents in all States of Brazil, from 1980 to 2005. We used the Brazilian Army's database, with 8,989,508 males 17-19 years of age. We defined overweight as body mass index (BMI) from $25 \mathrm{~kg} / \mathrm{m}^{2}$ to $29,9 \mathrm{~kg} / \mathrm{m}^{2}$ and obesity as $B M I \geq 30 \mathrm{~kg} / \mathrm{m}^{2}$. The analysis showed an increasing trend in the prevalence of overweight and obesity in all States of Brazil for the period studied. There was a three-fold increase in adolescents with overweight and a six-fold increase in obesity. The absolute numbers for overweight were higher than for obesity, but the rate of increase over the period was greater for obesity. Analyzing the trend in prevalence rates by fiveyear period, we observed that from 1980 to 2005, as the prevalence of overweight decreased, that of obesity increased in all States of Brazil.
\end{abstract}

Obesity; Overweight; Temporal Distribution; Adolescent
Vera Lucia de Vasconcelos Chaves 1 Eduardo Freese 1

Tiago Maria Lapa 1

Eduarda Ângela Pessoa Cesse 1

Ana Lucia Ribeiro de Vasconcelos 1

\section{Introdução}

A sucessão de mudanças, particularmente as ocorridas a partir da industrialização e da urbanização durante o século XX, acontece de forma distinta nos diversos continentes, e nos diferentes processos históricos de vários países e até mesmo entre regiões de um mesmo país, gerando estilos de vida próprios, com repercussões para o indivíduo e para as populações, especificamente no que diz respeito às alterações nos padrões de dieta e, conseqüentemente, nas condições de saúde 1,2,3,4.

Nesse contexto, o Brasil apresenta um mosaico em que coexistem as doenças de carência alimentar, tais como a desnutrição protéicocalórica, anemia ferropriva e avitaminoses, e as doenças de excesso, como as dislipidemias, o sobrepeso e a obesidade. Vale salientar que nas últimas décadas, a configuração deste quadro vem mudando, revelando uma significativa redução da desnutrição e uma acelerada ascensão da prevalência de obesidade. Os fatos demonstram um explícito processo de Transição Nutricional no país, fato este já documentado por diversos autores $5,6,7$.

Dessa forma o sobrepeso e a obesidade apresentam um enorme potencial de expansão tanto em sociedades desenvolvidas quanto nas em desenvolvimento e, por esse acelerado aumento, vêm atingindo proporções que podem ser consideradas como "epidêmicas" 8,9,10. Historicamen- 
te, isso nunca havia sido registrado com semelhante magnitude e, por estar associado a outros fatores de risco, é considerado um dos principais determinantes da alta prevalência de doenças crônicas não transmissíveis 8,9,11,12,13.

Segundo Marques-Lopes et al. 14, o excesso de peso é geralmente atenuado ou exacerbado pelos fatores ambientais como alimentação e exercício físico. Outras investigações sugerem que é o gasto energético e não a ingesta calórica, que difere entre as pessoas magras e obesas 15,16,17. Do ponto de vista epidemiológico, os determinantes ambientais são apontados como as principais causas de obesidade, uma vez que, nas últimas décadas, não ocorreram alterações substanciais nas características genéticas das populações, enquanto as mudanças nos seus hábitos foram relevantes $3,7,15,16,18,19,20$.

Diante desse quadro, a obesidade se posiciona como um dos importantes problemas de saúde pública na atualidade, acometendo todas as idades, sexo, raça e parcelas da população com diferentes condições sócio-econômicas 2,6,9,12.

Este artigo objetiva determinar a evolução espaço-temporal da prevalência do sobrepeso e da obesidade em adolescentes masculinos brasileiros, no período de 1980-2005, tendo em vista a constatação da carência de estudos semelhantes nesta faixa etária no Brasil.

\section{Métodos}

Foi realizado um estudo epidemiológico de prevalência e de tendência temporal, utilizando-se como fonte de informação o banco de dados do alistamento militar do Exército Brasileiro. O banco de dados foi cedido pelo Centro Integrado de Telemática do Exército Brasileiro (CITEX), sendo disponibilizado um CD contendo informações dos adolescentes inscritos para o serviço militar entre os anos de 1980 e 2005 em todo o Brasil.

Os registros existentes no banco de dados correspondem às informações de 30\% a 40\% dos alistados no Serviço Militar por estado, que foram selecionados por sorteio aleatório simples e encaminhados para as Juntas de Inspeção de Saúde, a fim de realizarem avaliação médicopsicológica para serem incorporados às Forças Armadas - Marinha, Exército e Aeronáutica. A obesidade não consta na lista das doenças que motivam a isenção definitiva dos conscritos para o Serviço Militar nas Forças Armadas.

A população de estudo foi de 8.989 .508 de adolescentes do sexo masculino, na faixa etária de 17-19 anos de idade, residentes em todos os estados do Brasil. A prevalência do sobrepeso e da obesidade foi quantificada a cada ano, de
1980-2005, permitindo verificar a tendência temporal desses agravos.

O índice de massa corporal (IMC) ou índice de Quetelet é obtido pela divisão do peso em quilogramas pela estatura em metros ao quadrado e, de acordo com a Organização Mundial da Saúde (OMS) 21, o peso dos adultos é classificado como normal $\left(\mathrm{IMC}=18,5-24,9 \mathrm{~kg} / \mathrm{m}^{2}\right)$, sobrepeso $\left(\mathrm{IMC}=25,0-29,9 \mathrm{~kg} / \mathrm{m}^{2}\right.$ ) e obesidade (IMC $\geq$ $\left.30 \mathrm{~kg} / \mathrm{m}^{2}\right)$.

As variáveis foram categorizadas em: dependentes (sobrepeso e obesidade, classificadas, considerando as recomendações da OMS 21); e independentes (relativas ao tempo, os anos de 1980-2005; relativas à idade, à faixa etária de 17 19 anos; relativas ao lugar, local de residência, ou seja, os estados do Brasil).

Foram padronizados os procedimentos na coleta dos dados antropométricos seguindo as técnicas recomendadas pela OMS 21.

Os cálculos foram realizados com o auxílio do programa SPSS, versão 10.0 (SPSS Inc., Chicago, Estados Unidos).

Considerando as recomendações da OMS 21, classificamos em quatro grupos: baixo peso; eutrófico; sobrepeso e obesidade; e excluímos do estudo os considerados baixo peso.

As prevalências do sobrepeso e da obesidade foram calculadas com os respectivos intervalos de 95\% de confiança (IC95\%), nos vinte e seis cortes transversais, para cada estado separadamente. A razão de prevalência foi calculada dividindo-se a prevalência final pela prevalência inicial, em cada estado e no Brasil.

Em seguida, foi realizado agrupamento por décadas a fim de analisar as mudanças ocorridas ao longo dos vinte e seis anos estudados.

Para a análise de tendência temporal do sobrepeso e da obesidade, foram realizados modelos de regressão linear simples, definidos como: $\mathrm{Y}=\alpha+\beta$ ano, sendo $\alpha$ o coeficiente médio no período analisado e $\beta$ o incremento (acréscimo ou decréscimo) médio no período. O R2 (Coeficiente de Determinação) corresponde à mensuração da força de relação linear. As conclusões foram tomadas a IC95\%.

Tendo em vista a existência de duas variáveis (sobrepeso e obesidade) indicando aumento de peso, foi construído um indicador composto (excesso de peso), valendo-se dos coeficientes de inclinação ("b”) para cada variável, e, com base na padronização de 0 a 1 para cada uma das Unidades da Federação (UF), foram obtidos valores com escore impessoal.

Para a elaboração do indicador composto, foi empregada inicialmente a metodologia de aplicação de escores, garantindo a ordenação das UF segundo uma única escala de valores 22,23 . 
Após o cálculo das médias para cada UF, os escores foram listados segundo uma escala crescente de valores dos indicadores, em valores de 0 a 1 , onde " 1 " indica os maiores e " 0 " os menores, necessários para o cálculo dos escores. Posteriormente, foram utilizados os quartis da distribuição do indicador como pontos de corte.

Com o intuito de obter visão espacial e analisar a evolução do problema no tempo, esta mesma análise foi realizada por qüinqüênio.

Os resultados da análise da prevalência por qüinqüênio foram apresentados em Mapas, com uma escala de cor crescente, de acordo com o aumento dos valores encontrados para o sobrepeso e para obesidade separadamente.

Como provável limitação metodológica, é importante pautar que apesar de terem sido utilizados dados secundários para o estudo, de modo que erros nas medidas antropométricas podem ter ocorrido em decorrência de falhas humanas ou do instrumental utilizado, por variações inter e intra-examinador, acredita-se que em se tratando de um estudo populacional, de uma amostra representativa, os possíveis erros existentes não sejam relevantes, especialmente por serem aleatórios, afetando todos os grupos igualmente.

Apesar de o estudo ter sido realizado com dados secundários, e, portanto sem um valor direto de diagnóstico para a pessoa sujeita à pesquisa, dispensando autorização prévia dos participantes, as recomendações contidas na Declaração de Helsinki foram respeitadas. O projeto de pesquisa foi encaminhado ao Comitê de Ética em Pesquisa do Centro de Pesquisa Aggeu Magalhães, Fundação Oswaldo Cruz (CPqAM/FIOCRUZ) e aprovado em 13 de setembro de 2006.

\section{Resultados}

A tendência temporal de prevalência do sobrepeso e da obesidade em adolescentes masculinos brasileiros no período de 1980-2005 foi crescente no Brasil, com uma razão de prevalência de 3,0 para o sobrepeso e de 6,0 para a obesidade (Tabela 1).

Analisando a velocidade de ganho por décadas no Brasil, percebe-se que, em relação ao sobrepeso, houve uma desaceleração progressiva, apresentando uma Razão de Prevalência na Primeira Década $(\mathrm{RP} 1)=1,7$, na segunda uma $\mathrm{RP} 2=1,6$ e, na terceira uma RP3 = 1,0. Em relação à obesidade, observou-se que a velocidade de incremento ocorreu da primeira para a segunda década. No entanto, da segunda para a terceira década, verificamos importante desaceleração desse crescimento $(\mathrm{RP} 1=2,0, \mathrm{RP} 2=2,3$ e RP3 = 1,2). Foi constatado também, que os valores ab- solutos de sobrepeso foram maiores que os da obesidade, porém, ao analisar a prevalência no início e no final do estudo (1980 e 2005), encontrou-se que a velocidade de ganho em vinte e seis anos foi maior para obesidade (Tabela 1).

A análise de tendência linear das curvas de crescimento mostrou que os valores do sobrepeso e da obesidade foram positivos, com a inclinação da reta "b" crescente no período estudado e um aumento médio da prevalência do sobrepeso de 0,35 e da obesidade de 0,06, como podemos ver nas equações: sobrepeso ( $\mathrm{y}=0,3489$ $x 3,4791)$ e obesidade ( $y=0,0604 \times 0,1612)$. A mensuração da força de relação linear expressa em $\mathrm{R}^{2}$ é elevada, próximos de um (sobrepeso = 0,9494 e obesidade $=0,9051$ ), levando a concluir que o crescimento da prevalência de sobrepeso e obesidade é fortemente explicado pelo modelo utilizado.

Os valores de prevalência do sobrepeso e da obesidade em cada estado do Brasil, quando analisados separadamente no período considerado, também foram crescentes. Em relação ao sobrepeso todos os valores de "b" são positivos (valor de $\mathrm{p}<0,005$ ). O estado que apresentou maior velocidade de ganho foi o do Rio Grande do Norte $(b=0,577)$, seguido dos Estados do Rio de Janeiro $(b=0,433)$, Espírito Santo $(b=0,429)$ e Pernambuco $(b=0,408)$. As menores velocidades de ganho foram observadas no Estado do Amapá $(b=0,191)$, e no Estado do Maranhão $(b=0,198)$. O coeficiente de correlação (R²) foi expressivo na grande maioria dos estados, com exceção de Roraima $\left(R^{2}=0,429\right)$, sendo mais intenso no Estado de Santa Catarina $\left(\mathrm{R}^{2}=0,964\right)$ (Tabela 2$)$.

Em relação à obesidade, os valores de "b" também foram positivos na grande maioria dos estados analisados. A exceção foi o Estado de Roraima, que apresentou valor negativo $(b=$ $-0,374)$, com declínio na sua curva. Na grande maioria dos estados, o crescimento apresentou significância estatística (valor de $\mathrm{p}<0,005$ ), com exceção do Estado de Sergipe (valor de p = 0,045) (Tabela 2).

Com o objetivo de demonstrar os estados que apresentam as melhores e piores condições de sobrepeso e obesidade, foram comparados os valores de prevalência de cada um separadamente com a prevalência média do Brasil, classificando-se os valores obtidos por quartil. Observa-se que os estados em melhores condições encontram-se nas regiões Norte e Centro-oeste, e os que estão em piores condições, nas regiões Sul e Sudeste. No Nordeste, são encontrados os estados com as maiores e com as menores prevalências (Tabela 2).

Ao analisar a evolução da prevalência do sobrepeso e da obesidade por qüinqüênio, pode- 
Tabela 1

Prevalência do sobrepeso e da obesidade por décadas, em adolescentes masculinos de 17-19 anos de idade. Brasil, $1980-2005$.

\begin{tabular}{|c|c|c|c|c|c|}
\hline Ano & $\mathrm{n}$ & Sobrepeso (\%) & IC95\% & Obesidade (\%) & IC95\% \\
\hline 1980 & 310.973 & 4,0 & $3,99988-4,00012$ & 0,3 & $0,29997-0,30003$ \\
\hline 1981 & 332.474 & 4,5 & $4,49988-4,50012$ & 0,6 & $0,59995-0,60005$ \\
\hline 1982 & 432.578 & 4,8 & $4,79990-4,80010$ & 0,6 & 0,59997-0,60003 \\
\hline 1983 & 436.636 & 4,6 & 4,59991-4,60009 & 0,5 & $0,49997-0,50003$ \\
\hline 1984 & 409.372 & 4,2 & $4,19990-4,20010$ & 0,5 & $0,49997-0,50003$ \\
\hline 1985 & 434.826 & 4,6 & $4,59991-4,60009$ & 0,5 & $0,49997-0,50003$ \\
\hline 1986 & 347.421 & 7,1 & 7,09986-7,10014 & 0,5 & $0,49996-0,50004$ \\
\hline 1987 & 314.005 & 6,2 & $6,19985-6,20015$ & 0,5 & 0,49996-0,50004 \\
\hline 1988 & 351.306 & 6,0 & $5,99987-6,00013$ & 0,5 & $0,49996-0,50004$ \\
\hline 1989 & 336.393 & 6,8 & $6,79985-6,80015$ & 0,6 & $0,59996-0,60004$ \\
\hline RP1 & 3.705 .984 & 1,7 & & 2,0 & \\
\hline 1990 & 312.185 & 6,6 & 6,59984-6,60016 & 0,6 & 0,59995-0,60005 \\
\hline 1991 & 270.895 & 8,0 & $7,99980-8,00020$ & 0,8 & $0,79994-0,80006$ \\
\hline 1992 & 327.475 & 7,9 & 7,89984-7,90016 & 0,7 & $0,69995-0,70005$ \\
\hline 1993 & 335.110 & 8,5 & $8,49984-8,50016$ & 0,9 & $0,89994-0,90006$ \\
\hline 1994 & 309.870 & 8,4 & $8,39982-8,40018$ & 0,9 & $0,89994-0,90006$ \\
\hline 1995 & 392.114 & 9,9 & $9,89985-9,90015$ & 1,2 & $1,19995-1,20005$ \\
\hline 1996 & 364.116 & 10,4 & $10,39984-10,40016$ & 1,3 & $1,29994-1,30006$ \\
\hline 1997 & 327.789 & 10,1 & $10,09982-10,10018$ & 1,2 & $1,19993-1,20007$ \\
\hline 1998 & 333.005 & 10,9 & $10,89982-10,90018$ & 1,4 & $1,39993-1,40007$ \\
\hline 1999 & 346.069 & 11,0 & $10,99982-11,00018$ & 1,4 & $1,39993-1,40007$ \\
\hline RP2 & 3.318 .628 & 1,6 & & 2,3 & \\
\hline 2000 & 261.282 & 11,3 & $11,29976-11,30024$ & 1,5 & $1,49991-1,50009$ \\
\hline 2001 & 281.792 & 11,2 & $11,19978-11,20022$ & 1,6 & $1,59991-1,60009$ \\
\hline 2002 & 241.135 & 11,4 & $11,39974-11,40026$ & 1,6 & $1,59990-1,60010$ \\
\hline 2003 & 241.593 & 11,0 & $10,99975-11,00025$ & 1,6 & $1,59990-1,60010$ \\
\hline 2004 & 270.708 & 11,3 & 11,29977-11,30023 & 1,7 & $1,69991-1,70009$ \\
\hline 2005 & 449.361 & 12,0 & $11,99986-12,00014$ & 1,8 & $1,79994-1,80006$ \\
\hline RP3 & 1.745 .871 & 1,0 & & 1,2 & \\
\hline RPF & 8.770 .483 & 3,0 & & 6,0 & \\
\hline
\end{tabular}

IC95\%: intervalo de 95\% de confiança; RP1: razão de prevalência na primeira década; RP2: razão de prevalência na segunda década; RP3: razão de prevalência na terceira década; RPF: razão de prevalência final.

mos verificar que à medida que a prevalência do sobrepeso diminuiu, a prevalência da obesidade aumentou nos estados brasileiros, considerando o período de 1980-2005 (Figura 1 e 2).

Vale destacar o decréscimo dos valores de eutróficos achados pela análise de tendência linear que revelam valores de " $b$ " negativos, indicando que a curva foi decrescente no decorrer dos anos, com valores estatisticamente significativos (valor de $\mathrm{p}<0,005$ ) (Tabela 2).

\section{Discussão}

O presente estudo analisou a evolução espaçotemporal da prevalência do sobrepeso e da obesidade, no período de 1980-2005. Para tanto, adotou critérios antropométricos em uma amostra aleatória, uniforme e abrangente, composta de adolescentes masculinos na faixa etária de 17 19 anos de idade, alistados no Serviço Militar do Exército Brasileiro de todos os estados do país.

Torna-se necessário enfatizar que para diversos autores 24,25,26,27,28,29 a adoção de critério antropométrico para investigar a obesidade é muito mais complexo e difícil em adolescentes do que em outros grupos etários. Para Veiga et al. 27, 
Análise de regressão linear simples da prevalência do eutrófico, do sobrepeso e da obesidade, segundo os estados do Brasil, 1980-2005.

\begin{tabular}{|c|c|c|c|c|c|c|c|c|c|}
\hline \multicolumn{4}{|c|}{ Eutrófico } & \multicolumn{3}{|c|}{ Sobrepeso } & \multicolumn{3}{|c|}{ Obesidade } \\
\hline UF & b & $\mathbf{R}^{2}$ & Valor de $p$ & b & $\mathbf{R}^{2}$ & Valor de $p$ & b & $\mathbf{R}^{2}$ & Valor de $p$ \\
\hline RN & $-0,659$ & 0,913 & $<0,001$ & 0,577 & 0,916 & $<0,001$ & 0,082 & 0,772 & $<0,001$ \\
\hline RJ & $-0,509$ & 0,913 & $<0,001$ & 0,433 & 0,915 & $<0,001$ & 0,076 & 0,872 & $<0,001$ \\
\hline ES & $-0,499$ & 0,936 & $<0,001$ & 0,429 & 0,931 & $<0,001$ & 0,071 & 0,831 & $<0,001$ \\
\hline RS & $-0,495$ & 0,907 & $<0,001$ & 0,395 & 0,889 & $<0,001$ & 0,100 & 0,879 & $<0,001$ \\
\hline PE & $-0,478$ & 0,929 & $<0,001$ & 0,408 & 0,934 & $<0,001$ & 0,071 & 0,856 & $<0,001$ \\
\hline SC & $-0,465$ & 0,966 & $<0,001$ & 0,396 & 0,964 & $<0,001$ & 0,069 & 0,919 & $<0,001$ \\
\hline SE & $-0,442$ & 0,642 & $<0,001$ & 0,391 & 0,757 & $<0,001$ & 0,051 & 0,163 & 0,045 \\
\hline CE & $-0,441$ & 0,819 & $<0,001$ & 0,375 & 0,831 & $<0,001$ & 0,065 & 0,646 & $<0,001$ \\
\hline PR & $-0,422$ & 0,952 & $<0,001$ & 0,344 & 0,942 & $<0,001$ & 0,078 & 0,933 & $<0,001$ \\
\hline $\mathrm{RO}$ & $-0,390$ & 0,78 & $<0,001$ & 0,285 & 0,782 & $<0,001$ & 0,080 & 0,718 & $<0,001$ \\
\hline GO & $-0,387$ & 0,911 & $<0,001$ & 0,341 & 0,918 & $<0,001$ & 0,046 & 0,616 & $<0,001$ \\
\hline MS & $-0,385$ & 0,94 & $<0,001$ & 0,312 & 0,946 & $<0,001$ & 0,073 & 0,694 & $<0,001$ \\
\hline MG & $-0,384$ & 0,923 & $<0,001$ & 0,329 & 0,926 & $<0,001$ & 0,055 & 0,818 & $<0,001$ \\
\hline PA & $-0,379$ & 0,813 & $<0,001$ & 0,328 & 0,805 & $<0,001$ & 0,051 & 0,742 & $<0,001$ \\
\hline SP & $-0,367$ & 0,729 & $<0,001$ & 0,316 & 0,669 & $<0,001$ & 0,051 & 0,717 & $<0,001$ \\
\hline$A C$ & $-0,355$ & 0,68 & $<0,001$ & 0,265 & 0,675 & $<0,001$ & 0,068 & 0,732 & $<0,001$ \\
\hline RR & $-0,345$ & 0,345 & 0,002 & 0,215 & 0,429 & 0,001 & $-0,374$ & 0,524 & 0,002 \\
\hline $\mathrm{PI}$ & $-0,336$ & 0,818 & $<0,001$ & 0,289 & 0,814 & $<0,001$ & 0,047 & 0,719 & $<0,001$ \\
\hline$A L$ & $-0,333$ & 0,684 & $<0,001$ & 0,288 & 0,68 & $<0,001$ & 0,045 & 0,563 & $<0,001$ \\
\hline PB & $-0,324$ & 0,695 & $<0,001$ & 0,296 & 0,684 & $<0,001$ & 0,028 & 0,585 & $<0,001$ \\
\hline MT & $-0,323$ & 0,819 & $<0,001$ & 0,287 & 0,837 & $<0,001$ & 0,036 & 0,468 & $<0,001$ \\
\hline AM & $-0,319$ & 0,55 & $<0,001$ & 0,238 & 0,619 & $<0,001$ & 0,053 & 0,613 & $<0,001$ \\
\hline TO & $-0,302$ & 0,738 & $<0,001$ & 0,243 & 0,676 & $<0,001$ & 0,058 & 0,484 & 0,001 \\
\hline DF & $-0,300$ & 0,728 & $<0,001$ & 0,271 & 0,734 & $<0,001$ & 0,029 & 0,342 & 0,002 \\
\hline BA & $-0,245$ & 0,808 & $<0,001$ & 0,214 & 0,814 & $<0,001$ & 0,031 & 0,734 & $<0,001$ \\
\hline AP & $-0,243$ & 0,546 & $<0,001$ & 0,191 & 0,512 & $<0,001$ & 0,052 & 0,414 & 0,001 \\
\hline MA & $-0,229$ & 0,612 & $<0,001$ & 0,198 & 0,589 & $<0,001$ & 0,030 & 0,613 & $<0,001$ \\
\hline
\end{tabular}

UF: Unidade de Federação; b: coeficiente de inclinação; R2: coeficiente de determinação.

Estados brasileiros: AC: Acre; AL: Alagoas; AM: Amazonas; AP: Amapá; BA: Bahia; CE: Ceará; DF: Distrito Federal; ES: Espírito Santo; GO: Goiás; MA: Maranhão; MG: Minas Gerais; MS: Mato Grasso do Sul; MT: Mato Grosso; PA: Pará; PB: Paraíba; PE: Pernambuco; PI: Piauí; PR: Paraná; RJ: Rio de Janeiro; RO: Rondônia; RR: Roraima; SC: Santa Catarina; SE: Sergipe; SP: São Paulo; RN: Rio Grande do Norte; RS: Rio Grande do Sul; TO: Tocantins.

a utilização do IMC durante a adolescência merece cautela por várias razões: o índice pode não depender da estimativa de gordura corporal; há diferentes estágios maturacionais individuais; composição corporal com modificação rápida e contínua, dentre outros. No entanto, Anjos 29 considera que o IMC ou índice de Quetelet, que tem seu uso amplamente disseminado na população, e cujo valor normal varia com idade e sexo, é considerado atualmente a melhor alternativa clínica para mensurar a adiposidade, sobretudo em estudos populacionais.

Mesmo considerando suas limitações, este foi o método que escolhemos para nosso estudo, por ser o proposto pela OMS 21 e o mais utilizado universalmente em estudos de base populacional 24,25,26,29,30.

Analisando as prevalências de sobrepeso e obesidade no Brasil, verificamos que a curva é ascensional, mostrando que a tendência temporal, no período analisado, foi crescente. Ao observarmos os valores absolutos das prevalências de obesidade, encontramos que os mesmos são pequenos, mas, ao analisarmos sua velocidade de incremento, percebemos que em três décadas este valor aumentou 6,0 vezes. Em relação aos valores absolutos das prevalências de sobrepeso, verificamos que eles são maiores que os de obesidade. Sua velocidade de ganho, todavia, não seguiu as mesmas proporções, e no período 

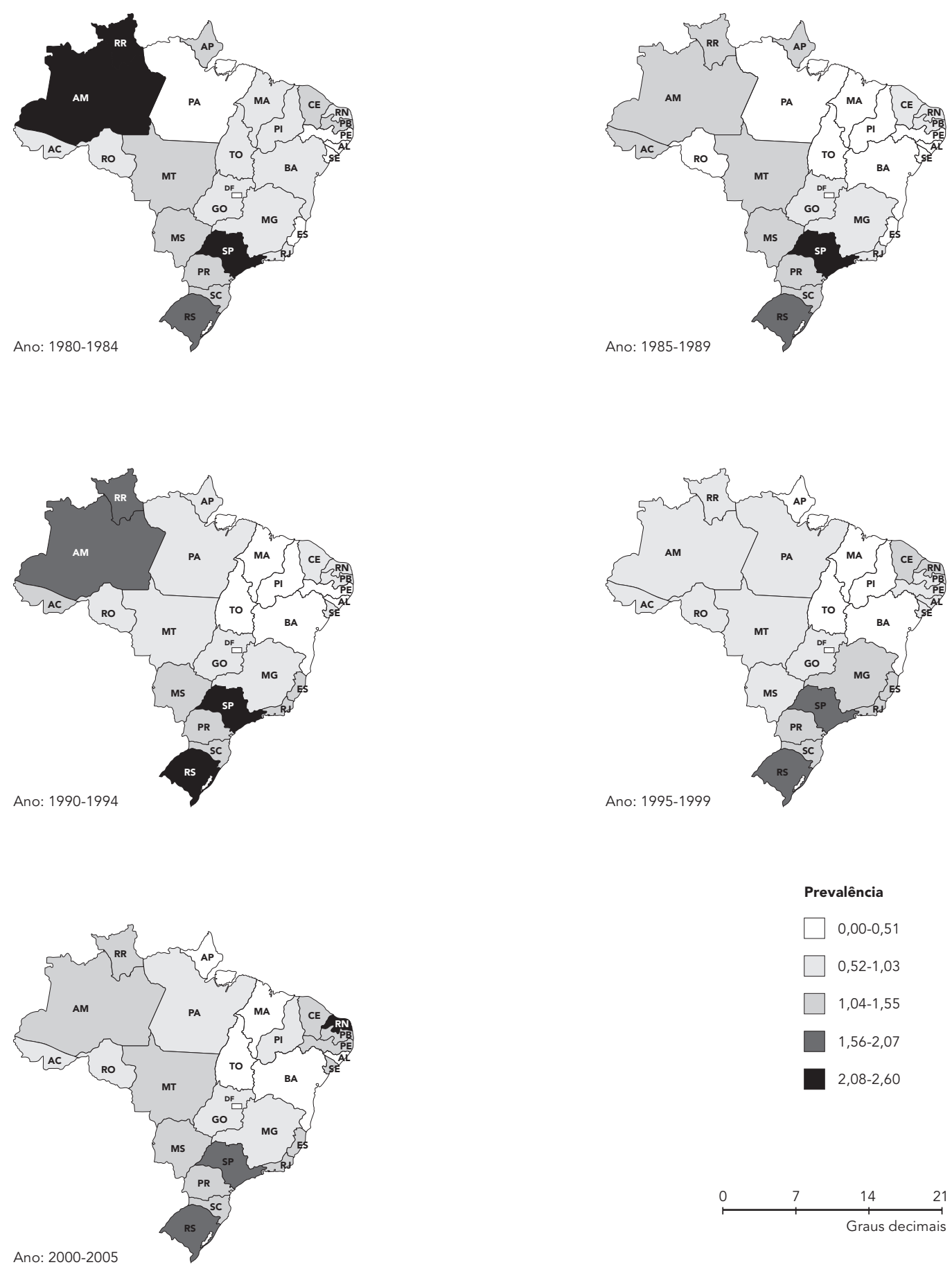

Estados brasileiros: AC: Acre; AL: Alagoas; AM: Amazonas; AP: Amapá; BA: Bahia; CE: Ceará; DF: Distrito Federal; ES: Espírito Santo; GO: Goiás; MA: Maranhão; MG: Minas Gerais; MS: Mato Grasso do Sul; MT: Mato Grosso; PA: Pará; PB: Paraíba; PE: Pernambuco; PI: Piauí; PR: Paraná; RJ: Rio de Janeiro; RO: Rondônia; RR: Roraima; SC: Santa Catarina; SE: Sergipe; SP: São Paulo; RN: Rio Grande do Norte; RS: Rio Grande do Sul; TO: Tocantins. 

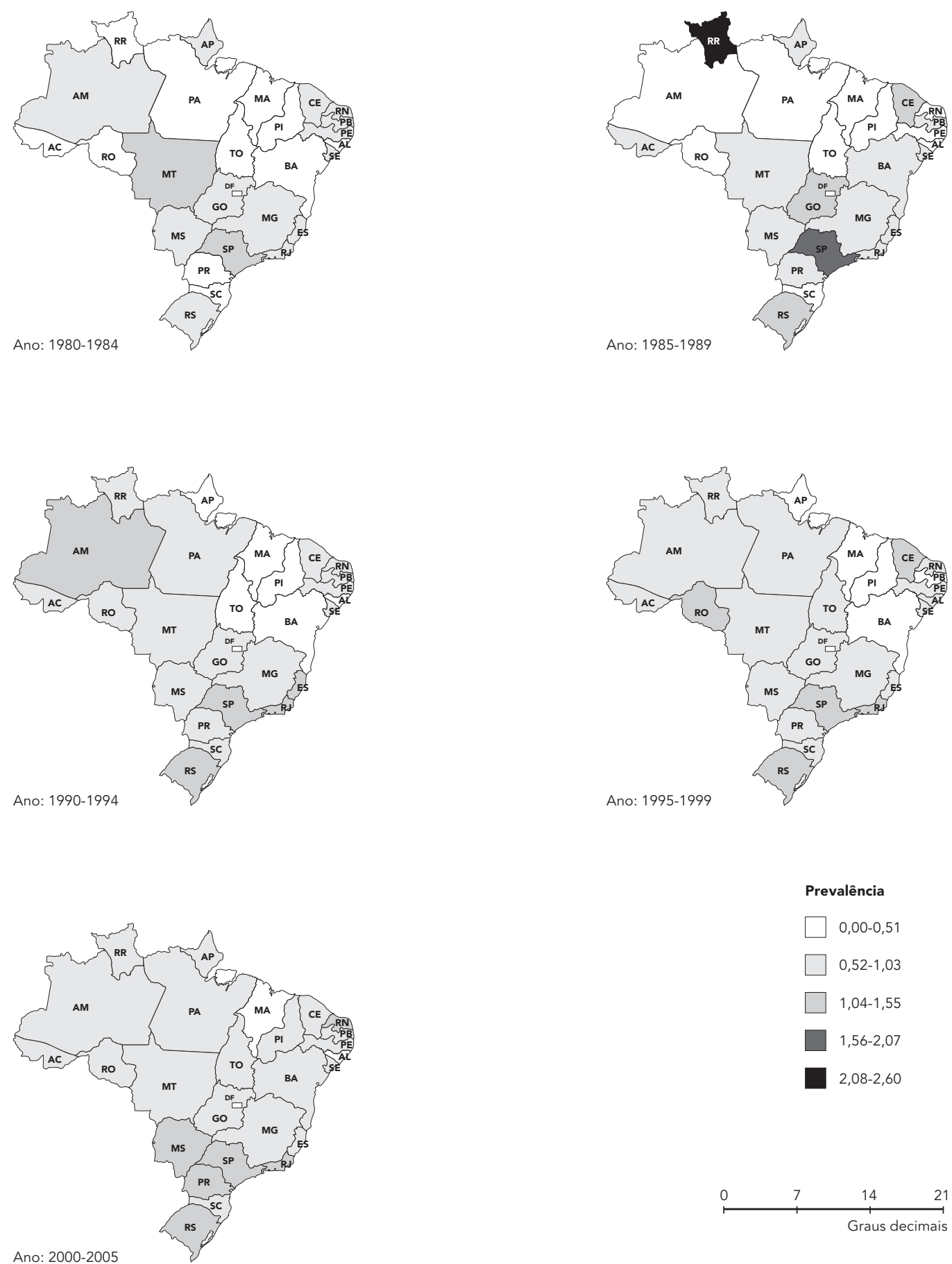

Ano: 1995-1999

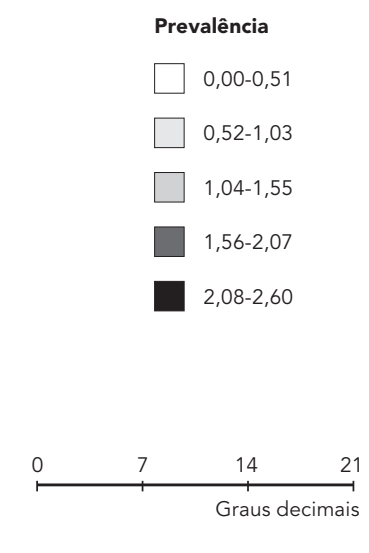

Estados brasileiros: AC: Acre; AL: Alagoas; AM: Amazonas; AP: Amapá; BA: Bahia; CE: Ceará; DF: Distrito Federal; ES: Espírito Santo; GO: Goiás; MA: Maranhão; MG: Minas Gerais; MS: Mato Grasso do Sul; MT: Mato Grosso; PA: Pará; PB: Paraíba; PE: Pernambuco; PI: Piauí; PR: Paraná; RJ: Rio de Janeiro; RO: Rondônia; RR: Roraima; SC: Santa Catarina; SE: Sergipe; SP: São Paulo; RN: Rio Grande do Norte; RS: Rio Grande do Sul; TO: Tocantins. 
de análise o aumento foi de 3,0 vezes (Tabela 1). Essa tendência epidemiológica foi observada igualmente em todos os estados do Brasil, onde verificamos que todas as curvas de sobrepeso e obesidade foram ascendentes (Tabela 2).

Tendência de crescimento do sobrepeso e da obesidade também foi observada em outros estudos. Neutzling et al. 31 , em um estudo populacional com adolescentes brasileiros na faixa etária de 10-19 anos de idade, de ambos os sexos, utilizando para diagnóstico o cálculo do IMC no percentil 85 e 95, encontraram prevalências de sobrepeso e obesidade mais baixas $(7,7 \%)$ que as apresentadas em inquéritos nacionais de outros países. Os autores, ao compararem seus achados com os resultados encontrados nos Estados Unidos e na Arábia Saudita, constataram que eles correspondem a três vezes menos. Enfatizaram, contudo, que este quadro é dinâmico e que o Brasil está sofrendo uma transição nutricional, em que a obesidade está aumentando de forma alarmante.

$\mathrm{Na}$ análise por décadas, observamos que o declínio do incremento do sobrepeso e o aumento do incremento da obesidade, ocorridos simultaneamente da primeira para segunda década, revelam que houve um agravamento da situação clínica dos adolescentes no período. No entanto, na terceira década ocorreu uma desaceleração do incremento tanto para o sobrepeso quanto para a obesidade. Vale destacar a maior velocidade de ganho na última década do período analisado, quando a prevalência do sobrepeso no Brasil aumentou de $4 \%$ (1980) para $12 \%$ (2005) e a prevalência da obesidade, de $0,3 \%$ (1980) para 1,8\% (2005) (Tabela 1).

Halpern 10 e Mondini \& Monteiro 32, em inquéritos nutricionais realizados no Brasil nas três últimas décadas, verificaram a existência de uma tendência de aumento do número de obesos em ambos os sexos. Esses autores revelaram que entre 1974 e 1989 a proporção de brasileiros com excesso de peso aumentou de $21 \%$ para $32 \%$. Que as maiores prevalências foram observadas no Sul do país, com valores semelhantes e até mesmo superiores à de países desenvolvidos. Esses autores demonstraram ainda que a evolução da ocorrência neste mesmo período em relação ao sexo dobrou entre os homens (de $2,4 \%$ para $4,8 \%$ ), e que na população feminina, o aumento da obesidade também foi importante, passando de $7 \%$ para $12 \%$. Esses autores mostraram, por fim, que a evolução da obesidade foi ascendente e relativamente uniforme no Nordeste e Sudeste, para homens e mulheres em todas as faixas etárias.

Veiga et al. 27 encontraram que a prevalência de sobrepeso para os meninos adolescentes é maior do que para obesidade; e que a prevalência de obesidade em meninas tem sido maior que o sobrepeso.

Além dos já citados, outros estudos de grande abrangência apontam a crescente tendência de aumento nas prevalências de sobrepeso e obesidade. Dados da Pesquisa sobre Padrões de Vida (PPV), coletados em 1997 pelo Instituto Brasileiro de Geografia e Estatística (IBGE) em convênio com o Banco Mundial 33, revelaram que a prevalência de sobrepeso em adolescentes brasileiros variou entre $1,7 \%$, no Nordeste, e $4,2 \%$, no Sudeste. E que a prevalência da obesidade em adolescentes variou entre $6,6 \%$ e $8,4 \%$, nas regiões Nordeste e Sudeste, respectivamente.

Estudo de prevalência realizado pela Organização Pan-Americana da Saúde (OPAS) 7 revelou que aproximadamente $6 \%$ da população brasileira apresenta obesidade (1,5\% na Região Nordeste e $4,2 \%$ na Região Sul).

Estudo realizado pelo Ministério da Saúde do Brasil 33 revelou que a prevalência de adultos com sobrepeso correspondia a 27 milhões $(32 \%$ da população), e com obesidade a 6,8 milhões ( $8 \%$ da população), e a prevalência nos adolescentes foi estimada em $20 \%$, demonstrando, assim, a preocupante magnitude do problema.

Os resultados do presente estudo revelam que os maiores incrementos observados em relação ao sobrepeso nos 26 anos estudados, foram vistos em três estados do Nordeste (Piauí, Sergipe e Rio Grande do Norte) e, em relação à obesidade, o maior ganho também ocorreu no Nordeste (Piauí). Esses estados do Brasil são historicamente menos favorecidos sócio-economicamente, o que pode sugerir um possível déficit estatural secundário a uma subnutrição nos primeiros cinco anos de vida, comprometendo o crescimento desses jovens. Na adolescência, esse fato acarretaria um maior aporte calórico-protéico, justificando o acúmulo de gordura com repercussão no valor do IMC. Essa hipótese encontrase respaldada nos resultados de outros estudos, nos quais os autores também relatam déficit de peso e estatura no Nordeste do Brasil 18,34.

Da mesma forma, os resultados da Pesquisa Nacional sobre Saúde e Nutrição (PNSN) 18 informam que $31 \%$ das crianças brasileiras menores de cinco anos apresentavam alguma forma de desnutrição, e as maiores taxas foram encontradas no Nordeste. O déficit mais freqüente apareceu na relação altura/idade, indicando predomínio da desnutrição crônica. Esses resultados revelam também que, aos 18 anos de idade, os jovens das regiões Norte e Nordeste, cujas famílias possuem renda até meio salário mínimo per capita, têm $13 \mathrm{~cm}$ a menos que a população de referência, enquanto os jovens da Região Sul têm $7 \mathrm{~cm}$ a menos. 
Oliveira et al. 34 disseram que as crianças brasileiras estão apresentando baixa estatura, sem, contudo, apresentarem magreza excessiva, fato que pode ser causado pelo uso de uma alimentação desequilibrada. O déficit estatural é contínuo ao longo de todo o período de crescimento, gerando altas prevalências de "nanicos" entre os adultos jovens. Segundo esses autores, calcula-se que um em cada cinco brasileiros de 20-26 anos tem altura inferior ao mínimo aceitável para sua idade e que a maior gravidade deste problema se encontra mais uma vez nas regiões Norte e Nordeste e nos grupos sociais de baixa renda.

Achados semelhantes foram divulgados pela OMS 21 ao reunir 79 inquéritos nacionais, realizados entre 1980 e 1992, nos países da África, Ásia e América Latina, cobrindo $87 \%$ da população total de crianças menores de cinco anos, ao verificar que os déficits de altura são mais comuns nos países em desenvolvimento.

Torna-se oportuno destacar a transição entre a desnutrição e a obesidade que já é uma realidade no país nas últimas décadas. Assim, a Pesquisa de Orçamentos Familiares de 2002-2003 do IBGE 35 detectou uma melhora nos indicadores antropométricos da população com menos de 20 anos em relação às medições feitas anteriormente. Isso indica que a desnutrição infantil diminuiu nos últimos 30 anos, especialmente entre aqueles que, historicamente, eram mais afetados pelo problema: as crianças das regiões Norte e Nordeste, e as das famílias com menores rendimentos. Essa pesquisa mostrou ainda que o excesso de peso já afeta um em cada cinco meninos residentes na Região Sul, Sudeste e Centro-oeste do Brasil.

Segundo Sichieri 6, tentar entender como uma população se torna obesa é uma tarefa difícil. O excesso de peso não é privilégio dos grupos mais favorecidos economicamente; pelo contrário, entre a população de baixa renda são observadas importantes prevalências de excesso de peso secundárias às limitações materiais importantes para a seleção de alimentos de menor teor energético, que são usualmente mais caros. Para Mendonça \& Anjos 16, tais aspectos se vinculam diretamente à renda das famílias e às possibilidades de gasto com alimentação, em particular associado ao valor sócio-cultural que os alimentos assumem em cada grupo social.

Considerando as abordagens acima apresentadas, verificamos que nas últimas décadas as pessoas estão consumindo maior quantidade de alimentos com grande densidade energética, com altos teores de açúcar e gorduras saturadas, e essa forma de nutrição, aliada a um sedentarismo crescente, está ocorrendo muito rapidamente nos países em desenvolvimento, que é o caso do Brasil.

Destacamos que o presente estudo corrobora com os resultados de estudos anteriores realizados no Brasil e em outros países, que demonstram tendência clara de aumento do número de obesos em ambos os sexos, em todas as idades e camadas sociais. Há, portanto, a necessidade de desenvolver estratégias preventivas, estimulando a reeducação alimentar e o aumento da atividade física, atingindo todas as camadas sociais.

\section{Resumo}

A análise de tendência temporal da obesidade revela riscos crescentes desse agravo em todo o mundo. Este artigo objetiva verificar e comparar as prevalências do sobrepeso e da obesidade em adolescentes masculinos de todos os estados do Brasil, de 1980-2005. Utilizamos o Banco de Dados do Exército Brasileiro com 8.989.508 adolescentes de 17-19 anos de idade. Consideramos como sobrepeso o índice de massa corporal (IMC) entre $25 \mathrm{~kg} / \mathrm{m}^{2}$ e 29,9kg/m², e obesidade como IMC $\geq 30 \mathrm{~kg} / \mathrm{m}^{2}$. Foi verificada tendência crescente da prevalência do sobrepeso e da obesidade, em todos os estados brasileiros, nos anos analisados. Nesse período, o número de adolescentes com sobrepeso aumentou em três vezes e com obesidade em seis vezes. Os valores absolutos de sobrepeso foram maiores que os da obesidade, porém a velocidade de ganho ao longo desse período foi maior para obesidade. Ao analisar a evolução das prevalências por qüinqüênio, observamos que de 1980-2005, à medida que a prevalência do sobrepeso diminuiu, a prevalência da obesidade aumentou em todos os estados brasileiros.

Obesidade; Sobrepeso; Distribuição Temporal; Adolescente 


\section{Colaboradores}

V. L. V. Chaves contribuiu na concepção e desenho do estudo, análise e interpretação dos dados, revisão do conteúdo intelectual, revisão final após contribuição dos co-autores, e encaminhamento da versão final para publicação. E. Freese e T. M. Lapa contribuíram substancialmente na concepção e desenho, análise e interpretação dos dados, revisão crítica do conteúdo intelectual, revisão e aprovação final da versão para publicação. A. L. R. Vasconcelos e E. A. P. Cesse colaboraram na análise e interpretação dos dados, revisão crítica do conteúdo intelectual e aprovação final da versão para publicação.

\section{Referências}

1. Freese EM, Fontbonne A. Transição epidemiológica comparada: modernidade, precariedade e vulnerabilidade. In: Freese EM, organizador. Epidemiologia, políticas e determinantes das doenças crônicas não transmissíveis no Brasil. Recife: Editora Universitária da UFPE; 2006. p. 17-46.

2. Monteiro CA, Conde WL. A tendência secular da obesidade segundo estratos sociais: Nordeste e Sudeste do Brasil, 1975-1989-1997. Arq Bras Endocrinol Metab 1999; 43:186-94.

3. Lopez FA, Escrivão MA, Oliveira FL, Taddei JA. Obesidade exógena na infância e na adolescência. J Pediatr (Rio J.) 2000; 76 Suppl 3:305-10.

4. Gutiérrez-Fisac JL, Regidor E, Lopez García E, Banegas Banegas JR, Rodríguez Artalejo F. La epidemia de obesidad y sus factores relacionados: el caso de España. Cad Saúde Pública 2003; 19 Suppl 1:101-10.

5. Monteiro CA, Mondini L, De Souza AL, Popkin BM. The nutrition transition in Brazil. Eur J Clin Nutr 1995; 49:105-13.

6. Sichieri R. Epidemiologia da obesidade. Rio de Janeiro: EdUERJ; 1998.

7. Pan American Health Organization. La obesidad en la pobreza: un nuevo reto para la salud pública. Washington DC: Pan American Health Organization; 2000. (Publicación Científica, 576).

\section{Agradecimentos}

Ao Exército Brasileiro, com destaque ao Centro Integrado de Telemática, ao Comando Militar do Nordeste e a todos os que colaboraram com a pesquisa.
8. Monteiro CA, Moura EC, Conde WL, Popkin BM. Socioeconomic status and obesity in adult populations in developing countries: a review. Bull World Health Organ 2004; 82:940-6.

9. Monteiro CA, Conde WL, Lu B, Popkin BM. Obesity and inequities in health in developing world. Int J Obes 2004; 28:1181-6.

10. Halpern A. A epidemia da obesidade. Arq Bras Endocrinol Metab 1999; 43:175-6.

11. Popkin BM. The nutrition transition and its health implications in lower income countries. Public Health Nutr 1998; 1:5-21.

12. Donald B. About obesity - source: International Obesity Task Force (IOTF). http://www.obesity. chaire.ulaval.ca/IOTF.htm (acessado em 25/Jul/ 2004).

13. Henry LL. Childhood obesity: what can be done to hel ptoday's youth? Pediatr Nurs 2005; 31:13-6.

14. Marques-Lopes I, Marti A, Moreno-Aliaga MJ, Martinez A. Aspectos genéticos da obesidade. Rev Nutr 2004; 17:327-38.

15. Willett W. Implication of total energy intake for epidemiologic studies. In: Willett W, editor. Nutritional epidemiology. New York: Oxford University; 1990. p. 245-71. 
16. Mendonça CP, Anjos LA. Aspectos das práticas alimentares e da atividade física como determinantes do crescimento do sobrepeso/obesidade no Brasil. Cad Saúde Pública 2004; 20:698-709.

17. Schonfeld-Warden N, Warden CH. Obesidade pediátrica: uma visão global da etiologia e do tratamento. Pediatr Clin North Am 1997; 2:343-66.

18. Instituto Nacional de Alimentação e Nutrição. Pesquisa Nacional sobre Saúde e Nutrição: perfil de crescimento da População Brasileira de 0 a 25 anos. Brasília: Instituto Nacional de Alimentação e Nutrição; 1990.

19. Anjos LA, Castro IRR, Engstrom EM, Azevedo AMF. Crescimento e estado nutricional em amostra probabilística de escolares no Município do Rio de Janeiro. Cad Saúde Pública 2003; 19 Suppl 1:171-9.

20. World Health Organization. Obesity: preventing and managing the global epidemic: report of a WHO Consultation on Obesity. Geneva: World Health Organization; 1997. (Technical Report Series, 894).

21. World Health Organization. Physical status: the use and interpretation of anthropometry: report of a WHO Expert Committee. Geneva: World Health Organization; 1995. (Technical Report Series, 854).

22. Fundo das Nações Unidas para a Infância. Municípios brasileiros: crianças e suas condições de sobrevivência. Censo Demográfico 1991. Brasília Fundo das Nações Unidas para a Infância; 1994.

23. Ximenes RAA, Martelli CMT, Souza WV, Lapa TM, Albuquerque MFM, Andrade ALSS, et al. Vigilância de doenças endêmicas em áreas urbanas: a interface entre mapas de setores censitários e indicadores de morbidade. Cad Saúde Pública 1999; 15:53-61.

24. Anjos LA, Veiga GV, Castro IRR. Distribuição dos valores do índice de massa corporal da população brasileira até 25 anos de idade. Rev Panam Salud Pública 1998;3:164-73.

25. Veiga GV, Vieira AC, Alvarez MM, Pereira RC. Índice de massa corporal na avaliação de sobrepeso e obesidade em crianças e adolescentes: concordâncias e controvérsias. Nutrire Rev Soc Bras Aliment Nutr 2004; 28:109-24.
26. Cole TJ, Bellizzi MC, Flegal KM, Dietz WD. Establishing a standard definition for child overweight and obesity worldwide: international survey. BMJ 2000; 320:1240-5.

27. Veiga GVD, Camacho P, Anjos LA. A comparison of distribution curves of body mass index from Brazil and the United States for assessing overweight and obesity in Brazilian adolescents. Rev Panam Salud Pública 2001; 10:79-85.

28. Chiara V, Sichieri R, Martins PD. Sensibilidade e especificidade de classificação de sobrepeso em adolescentes. Rev Saúde Pública 2003; 37:226-31.

29. Anjos LA. Obesidade e saúde pública. Rio de Janeiro: Editora Fiocruz; 2006.

30. Lazarus R, Baur L, Webb K, Blyth F. Body mass index in screening for adiposity in children and adolescents: systematic evaluation using receiver operating characteristic curves. Am J Clin Nutr 1996; 63:500-6.

31. Neutzling MB, Taddei JA, Rodrigues EM, Sigulem DM. Overweight and obesity in Brazilian adolescents. Int J Obes 2000; 24:869-74.

32. Mondini L, Monteiro CA. Relevância epidemiológica da desnutrição e da obesidade em distintas classes sociais: métodos de estudo e aplicação à população brasileira. Rev Bras Epidemiol 1998; 1:28-39.

33. Organización Panamericana de la Salud. La respuesta de salud pública a las enfermedades crónicas. Washington DC: Organización Panamericana de la Salud; 2002.

34. Oliveira JED, Cunha SFC, Marchini JSA. A desnutrição dos pobres e dos ricos: dados sobre a alimentação no Brasil. São Paulo: Sarvier Editora; 1996.

35. Instituto Brasileiro de Geografia e Estatística. Pesquisa de Orçamentos Familiares (POF) 2002-2003: antropometria e análise do estado nutricional de crianças e adolescentes no Brasil. Rio de Janeiro: Instituto Brasileiro de Geografia e Estatística; 2006.

Recebido em 16/Abr/2009

Versão final reapresentada em 20/Abr/2010

Aprovado em 03/Mai/2010 